\title{
GLOBAL THEOREMS IN RIEMANNIAN GEOMETRY
}

\section{B. ALLENDOERFER}

1. Introduction. I come to you today disguised as a missionary in an effort to interest you in the present day activities of differential geometers. The chief stimulus to differential geometry in the present century has been the General Theory of Relativity which is written in the language of Riemannian Geometry. Much of the effort of differential geometers in the twenties and thirties was directed toward generalizations of Riemannian Geometry which might lead to the elusive Unified Field Theory. With the virtual exhaustion of efforts in this direction several leading American differential geometers turned their attention to other fields of mathematics, others retired from active research, and the study of differential geometry in this country dropped to its lowest point in many years.

As this situation was deteriorating at home, a new trend in differential geometry was taking root abroad, namely the study of differential geometry "in the large." The general problem proposed was to develop relationships between the local differential properties of a space and its topologic structure as a whole. Leaders in this movement were H. Hopf and his pupils in Switzerland, de Rham in France, and Hodge in England. Contributions were made by various writers in the United States including Myers, Chern, Weil, and Allendoerfer; and the field has now matured to a point at which its general scope can be outlined and its major problems enumerated. It is now an active frontier in mathematics, ripe for development, and full of interest to mathematicians in numerous branches of our subject. Furthermore its investigation raises new questions in ordinary differential geometry, and these give promise of providing the stimulus needed to revive activity in differential geometry throughout this country.

Time is too short today to give a systematic survey of this field $;^{1}$ so I shall sketch the status of some of the major problems and give details of several topics which should serve as illustrations of the methods employed and the type of results that can be obtained.

2. The main problems. The raw material of the subject is a dif-

An address delivered before the New York meeting of the Society on October 25, 1947 , by invitation of the Committee to Select Hour Speakers for Eastern Sectional Meetings; received by the editors December 10, 1947.

${ }^{1}$ Such an article will appear in the author's detailed report on the session on differential geometry of the Princeton Bicentennial Conference on Mathematics. 
ferential manifold, namely a topological manifold defined in terms of neighborhoods each supplied with a coordinate system such that the coordinates of overlapping neighborhoods are differentiable functions of each other (with nonvanishing Jacobian) within the common region. The order of differentiability involved (usually called the class $\left.C^{k}\right)$ varies with the problem to be considered; but except for a few cases which require analyticity, fourth order partial derivatives are usually sufficient. On this manifold is imposed a Riemann metric

$$
d s^{2}=g_{\alpha \beta} d x^{\alpha} d x^{\beta}
$$

where $g_{\alpha \beta}$ is usually required to be positive definite. Such a manifold is called "complete" if it is complete in the usual sense; that is, if every Cauchy sequence converges; and it is proved that complete manifolds are also locally compact. Five main problems have arisen concerning such manifolds:

A. The problem of continuation. Here one is given an $n$-cell provided with an analytic Riemann metric, and the problem is to determine those complete manifolds (if any) to which this element can be continued. The chief contributions to this problem have been made by Myers [8]. ${ }^{2}$ He has shown that not every analytic Riemann element can be continued to a complete manifold and has given a number of necessary conditions and some sufficient conditions. A definitive set of necessary and sufficient conditions has yet to be found. Myers has also proved the following uniqueness theorem:

Theorem. Every n-dimensional Riemann element can be continued to at most one complete, simply-connected $n$-dimensional manifold $M$; that is, if two such continuations exist, they are isometric.

B. The problem of metrization. In a sense this is the inverse of the problem of continuation, namely: Given a manifold, what Riemann metrics (analytic or otherwise) can be used to metrize the entire manifold? The final solution of this question must await, among other things, the development of definitive topologic criteria for identifying homeomorphic manifolds. But even in the two-dimensional case where the topologic problem is solved, the problem of metrization is still open. Developments so far have been concerned (except in trivial cases) with necessary conditions. These seek to establish relationships between the differential invariants of the Riemann metric and the topology of the space. Connections of this sort have been made with the Euler-Poincaré characteristic, cohomology characteristic classes,

2 Numbers in brackets refer to the bibliography at the end of the paper. 
Betti numbers, and the fundamental group. Since I shall return to certain aspects of this problem, for the moment I shall pass on to a statement of the other problems.

C. The problem of imbedding. Here we are given a Riemann manifold, $M^{n}$, and ask the question: Is $M^{n}$ isometrically imbeddable in a Euclidean space? If so, what is the least dimensional Euclidean space for which the imbedding is possible? When the imbedding is possible, is it unique? Results in this field are most fragmentary, and consist chiefly of negative information and uniqueness theorems. For example, Hilbert has shown the impossibility of imbedding the hyperbolic plane in Euclidean three-space; but the fundamental questions stated above are unanswered for this manifold.

The uniqueness theorems state that an imbedding, if possible, is unique to within rigid motions or reflections. For two-dimensional surfaces in Euclidean three-space, Weyl [13] has shown that every convex surface is uniquely imbedded. For higher dimensions Beez [3] has shown that unicity of imbedding of $M^{n}$ in a Euclidean space of $n+1$ dimensions follows from the hypothesis that the second fundamental form of $M^{n}$ is of rank $\geqq 3$. His results were extended by Allendoerfer [1] to cover imbeddings in Euclidean spaces of arbitrary dimensions. These theorems, however, hold in the small as well as in the large, and the question of the unicity of imbedding in the large under more general hypotheses is still unanswered.

D. The problem of geodesics. In general terms this problem may be stated: What are the properties in the large of geodesics lying in a complete manifold? To be more specific consider the following question: If we have a geodesic, $C$, passing through a point $P$ of a complete manifold, how far can $C$ be extended from $P$ before it intersects itself or another geodesic through $P$, and how far along its path does $C$ measure a minimum distance from $P$ ? Considerable attention has been directed to the description of the loci of minimum and conjugate points of $P$. The results of these investigations are intended (among other things) to answer the question as to the maximum size of a normal coordinate system centered at a point $P$. Full results for $n=2$ have been obtained by Myers $[9,10]$, but a number of questions remain open for general values of $n$.

E. Metric theorems in the large. A number of theorems of differential geometry deal with properties of the whole of a curve or higher-dimensional subspace. Many of these were developed initially for plane curves, but when these are generalized to curves in Riemann manifolds it becomes essential to study the topology of these manifolds. Indeed without such study it is not possible to give an efficient or 
properly general statement of the theorem. As an example, I cite the well known theorem of Steiner on parallel curves. Let $C$ be a closed, convex plane curve and let $C_{\rho}$ be a curve outside $C$ parallel to it at a distance $\rho$; that is, $C_{\rho}$ is obtained by displacing the points of $C$ a distance $\rho$ along outward drawn normals to $C$. If $L$ is the length of $C$ and $L_{\rho}$ the length of $C_{\rho}$, Steiner showed that

$$
L_{p}=L+2 \pi \rho .
$$

The convexity hypothesis may be replaced by the equivalent requirement that no two normals to $C$ intersect outside $C$. However, when we turn to a right circular cylinder (which has the same local differential geometry as a plane), the theorem is no longer true. For a circular cross section of the cylinder and its parallels all have equal lengths. The difference is that the topology of a cylinder is not that of a plane. The theorem, however, is true on a cylinder if we restrict $C$ to curves which bound a finite area. Other theorems of this nature are the isoperimetric theorem, and the four vertex theorem.

3. The Kronecker index. Returning now to the problem of metrization, I wish to discuss certain aspects of this problem and to describe several tools which have proved useful in attacking it. The first of these is the Kronecker index of a vector field on a closed hypersurface $S^{n}$ of a manifold $M^{n+1}$. When $M^{n+1}$ is Euclidean this index takes the form:

$$
I=\frac{1}{\omega^{n}} \int_{B^{n}} D d x^{1} \cdots d x^{n}
$$

where

$$
D=\left|\begin{array}{ccc}
V^{1} & \cdots & V^{n+1} \\
\frac{\partial V^{1}}{\partial x^{1}} & \cdots & \frac{\partial V^{n+1}}{\partial x^{1}} \\
\cdots & \cdots & \cdots \\
\frac{\partial V^{1}}{\partial x^{n}} & \cdots & \frac{\partial V^{n+1}}{\partial x^{n}}
\end{array}\right|
$$

$x^{1}, \cdots, x^{n}$ are coordinates on $S^{n}$, and $V^{i}$ is a unit vector field in the Euclidean space having no singularities on $S^{n} . \omega^{n}$ is the area of an $n$ dimensional sphere. The useful properties of $I$ follow from the fact that it can be proved to be an integer; and this integer is shown to 
equal the algebraic sum of the orders of the singularities of $V^{i}$ within $S^{n}$.

For present purposes the most useful result is obtained when $n$ is even and $V^{i}$ is the unit normal vector field to $S^{n}$ in the Euclidean space. Then $I=\chi / 2$ where $\chi$ is the Euler-Poincare characteristic of $S^{n}$. Moreover in this case the integrand in (1) is expressible in terms of the determinants of the first and second fundamental forms of $S^{n}$, namely $g_{\alpha \beta}$ and $b_{\alpha \beta}$. Hence

$$
\frac{\chi}{2}=\frac{1}{\omega^{n}} \int_{S^{n}} \frac{\left|b_{\alpha \beta}\right|}{\left|g_{\alpha \beta}\right|}\left(\left|g_{\alpha \beta}\right|\right)^{1 / 2} d x^{1} \cdots d x^{n} .
$$

The ratio $\left|b_{\alpha \beta}\right| /\left|g_{\alpha \beta}\right|$ is called the "total curvature," $K_{T}$, of $S^{n}$ since it represents the product of the $n$ principal curvatures of the hypersurface. Since we have assumed that $n$ is even, $K_{T}$ can be expressed solely in terms of the intrinsic metric of $S^{n}$, namely in terms of $g_{\alpha \beta}$ and their derivatives. This generalization of Gauss' “Theorema Egregium" follows from the equations of Gauss:

$$
R_{\alpha \beta \gamma \delta}=b_{\alpha \gamma} b_{\beta \delta}-b_{\alpha \delta} b_{\beta \gamma}
$$

where $R_{\alpha \beta \gamma \sigma}$ is the Riemann tensor, an algebraic expression in $g_{\alpha \beta}$ and their derivatives. The result of this substitution is:

$$
K_{T}=\frac{R_{\alpha_{1} \alpha_{2} \beta_{1} \beta_{2}} R_{\alpha_{3} \alpha_{\varangle} \beta_{2} \beta_{4}} \cdots R_{\alpha_{n-1} \alpha_{n} \beta_{n-1} \beta_{n}} \epsilon^{\alpha_{1} \cdots \alpha_{n} \epsilon^{\beta_{1}} \cdots \beta_{n}}}{n ! 2^{n / 2}\left|g_{\alpha \beta}\right|}
$$

and hereafter $K_{T}$ will stand for this expression, even when we do not assume $S^{n}$ to be a subspace of any Euclidean space. Thus we have an integral relationship

$$
\int_{S^{n}} K_{T} g^{1 / 2} d x=\omega^{n} \cdot \frac{\chi}{2}
$$

between the intrinsic differential invariant $K_{T}$ and the topologic invariant $\chi$. Regarding $S^{n}$ as a given topologic manifold, this becomes a necessary condition upon the Riemann metrics which may be used to metrize it, and hence this is a contribution to the problem of metrization.

The validity of (5) so far has been established only under the assumption that $S^{n}$ is a hypersurface of a Euclidean space; but its intrinsic form suggests that it may be true for any closed differentiable manifold. This is indeed the case, and it should be remarked at this point that an excellent way of discovering relations like (5) is first to 
consider an imbedded manifold and then later to invent a new proof applicable to a general abstract manifold.

In order to establish the general validity of (5) and to illustrate further the Kronecker index, we shall follow a method due to Chern [5] in obtaining this result, which was first proved by AllendoerferWeil [2] in a more complicated fashion. It is well known that the Euler-Poincare characteristic of a closed manifold $M^{n}$ is equal to the algebraic sum of the orders of the singularities of an otherwise continuous vector field on $M^{n}$. By contrast to the normal vector field used above, these vectors are intrinsic, or tangent, vectors. It therefore appears desirable to find a generalization of (1) valid on Riemann manifolds. Following the usual procedure for making such generalizations, we replace the partial derivatives in (1) by covariant derivatives and introduce components of the Riemann tensor at appropriate places. However, there are so many ways in which the Riemann tensor can be introduced, that it is not easy to guess the correct form of the final answer. In order to do this systematically we consider for the moment only manifolds $M^{n}$ which are hypersurfaces of an $(n+1)$ dimensional Euclidean space. Using the method of tubes as developed by Allendoerfer-Weil we arrive at the following expression for $I$ :

$$
I=\lim _{S^{n-1} \rightarrow P} \int_{S^{n-1}} \phi d x^{1} \cdots d x^{n-1}
$$

where

$$
\phi=\frac{1}{\omega^{n}} \int_{-\pi / 2}^{\pi / 2} \Delta(\theta) d \theta
$$

where

$$
\Delta(\theta)=\mid \begin{array}{c|c}
V^{i} \text { columns } & 1 \text { row } \\
{\left[\cos \theta V_{, j}^{i}-\sin \theta g^{i k} b_{k j}\right] \frac{\partial y^{i}}{\partial x^{\alpha}}} & n-1 \text { rows. }
\end{array}
$$

In (6) and (7) $P$ is an isolated singularity of the unit vector field $V^{i}$; $S^{n-1}$ is an $(n-1)$-dimensional topologic sphere enclosing $P ; y^{i}$ are coordinates on $M^{n} ; x^{\alpha}$ are coordinates on $S^{n-1} ; g_{i j}$ and $b_{i j}$ are the first and second fundamental forms of $M^{n}$ relative to the Euclidean space in which it lies; $V_{{ }_{j}}^{d}$ is the covariant derivative of $V^{i}$ relative to the metric $g_{i j}$; and for convenience in the sequel the dimensions have 
been taken to be one lower than those of the corresponding formula (1).

At first sight $\phi$ does not appear to be intrinsic relative to $M^{n}$. However, after the integration the elements $b_{i k}$ appear only in second order minors of the matrix $\left\|b_{i k}\right\|$, and hence they can be expressed in terms of the Riemann tensor of $M^{n}$ by equations (3). The result of this substitution is a very complex expression which is omitted here (see Chern [5]). This expression has been derived on the assumption that $M^{n}$ is imbedded in a Euclidean space; but now that it has been invented, we use it to define $\phi$ for all manifolds $M^{n}$ whether imbedded or not. In all cases $I$ can be proved to be an integer which represents the order of the singularity of $V^{i}$ at $P$.

To find $\chi$ we then take the sum of the indices at each singularity, and the resulting sum can be expressed as an integral over $M^{n}$ by virtue of the generalized form of Stokes' Theorem which states that:

$$
\int_{B} \phi=\int_{R} d \phi
$$

where $R$ is a region of $M^{n}$ and $B$ is its boundary. It turns out that

$$
\begin{aligned}
d \phi & =\frac{2 K_{T}}{\omega^{n}} \\
& =0
\end{aligned}
$$

independently of the vector field $V^{i}$ from which we started. This establishes (5) in general. In this form (5) is a restricted case of the generalized Gauss-Bonnet Formula for Riemannian Polyhedra developed by Allendoerfer-Weil [2].

4. Problems growing out of the Gauss-Bonnet formula. As I stated at the outset, the development of such a result leads to new problems both in differential geometry and topology. The first of these concerns the analogous results for open manifolds. Cohn-Vossen [6] has proved that

$$
\int_{M^{n}} K_{T} g^{1 / 2} d x \leqq \omega^{n} \cdot \frac{\chi}{2}
$$

for two-dimensional open manifolds without boundary provided that the left-hand integral exists as an improper integral. The problem for $n$-dimensional open manifolds is unsolved. Its solution appears to require on the one hand a study of the topologic properties of vector fields defined on open manifolds, and on the other hand the addi- 
tional study of the differential geometry of the expression $\phi$. To illustrate one of the topologic difficulties I might mention that CohnVossen makes essential use of the fact that any finitely connected two-dimensional open manifold (without boundary) is homeomorphic to a closed manifold from which a finite number of points have been removed. No suitable generalization of this result for $n$ dimensions appears to be known.

The expression $\phi$ appears in the work of Allendoerfer-Weil and that of Chern, but its full meaning is yet to be uncovered. As a partial step in this direction I have noted that:

$$
A^{n-1} \equiv \int_{R^{n-1}} \phi d x^{1} \cdots d x^{n-1}
$$

is a proper definition of the solid angle of the vector field $V^{i}$ relative to a hypersurface $R^{n-1}$ of $M^{n}$. $R^{n-1}$ may be the whole of a closed hypersurface or may be any $(n-1)$-dimensional subset of a hypersurface. Because of the role which solid angle plays in physical field theories in ordinary space, it is likely that this expression may have applications to similar theories in general Riemann spaces.

Let the hypersurface $R^{n-1}$ have a boundary $B^{n-2}$ and let $\bar{R}^{n-1}$ be another hypersurface with the same boundary such that the vector field $V^{i}$ has no singularity in the volume enclosed by these two hypersurfaces. If the manifold $M^{n}$ is Euclidean, it is known that the solid angle of $V^{i}$ relative to $R^{n-1}$ is equal to that of $V^{i}$ relative to $\bar{R}^{n-1}$ and hence depends solely on the boundary $B^{n-2}$. In a curved Riemann space of even dimension this conclusion follows in general only if $K_{T}=0$. For the difference between these two solid angles is equal to the integral of $K_{T}$ over the enclosed volume. This means that an absolute notion of $(n-1)$-dimensional solid angle exists in an even-dimensional Riemann space when and only when $K_{T}=0$. When $n$ is odd, the two solid angles are equal without restriction. This situation is analogous to the known result that the angle between two vectors at different points in a Riemann space is independent of the path joining these points only when the Riemann space is flat; that is, when $R_{i j k l}=0$.

Levi-Civita has called a vector field "parallel" with respect to a curve, $C$, if $V_{, j}^{i} d y^{i} / d s=0$ along $C$ (whose equations are $y^{i}=f^{i}(s)$ ). When $n=2$,

$$
\phi=\frac{1}{2 \pi}\left|\begin{array}{cc}
V_{, j}^{1} & V^{2} \\
V_{, j}^{1} \frac{d y^{j}}{d s} & V_{, j}^{2} \frac{d y^{j}}{d s}
\end{array}\right|
$$


Since $V^{i}$ is assumed to be a unit vector, it follows that $V^{i}$ is "parallel" along $C$ if and only if $\phi=0$ along $C$. By way of generalization we may therefore say that a unit vector field on $M^{n}$ is parallel (of order $n-1$ ) relative to a hypersurface $R^{n-1}$ whenever $\phi=0$ on $R^{n-1}$. Further, just as absolute parallelism of Levi-Civita exists only when $R_{i j k l}=0$, absolute parallelism of order $n-1$ exists only when $n$ is odd, or (when $n$ is even) when $K_{T}=0$. A full discussion of this generalized type of parallelism together with the obvious intermediate cases must await another occasion. I mention it here chiefly as an illustration of the type of stimulus to ordinary Riemannian geometry which results from the study of problems in the large.

5. The nature of curvature. Let us now turn to the total curvature, $K_{T}$, itself. Although this quantity appears here and there in the literature, its study has been sadly neglected. Practically all the work on the "curvature" of Riemann spaces is concerned with the Riemann tensor $R_{i j k l}$ itself or with its contraction $R_{i j}$. Let me remind you that the common interpretation of $R_{i j k l}$ is in terms of the Gaussian twodimensional curvature of a geodesic two-dimensional surface associated with an arbitrary bi-vector. We have thus been limiting ourselves to an interpretation of the curvature of $n$-space in terms of our knowledge of two-space. It is time that we really set out to find some truly $n$-dimensional theorems not bound by this limitation. For example we can discuss the total curvature $K_{T}$ of the geodesic surface of $2 k$ dimensions associated with an arbitrary set of $2 k$ independent vectors $\lambda_{1}^{i}, \cdots, \lambda_{2 \mathbf{k}}^{i}$. Then we have the expression:

$$
K_{2 k} \equiv \frac{R_{r_{1} \cdots r_{2 k} ; p_{1} \cdots p_{2 k}} \lambda_{1}^{r_{1}} \cdots \lambda_{2 k}^{r_{2 k}} \lambda_{1}^{p_{1}} \cdots \lambda_{2 k}^{p_{2 k}}}{g_{r_{1}} \cdots r_{2 k} ; p_{1} \cdots p_{k 2} \lambda_{1}^{r} \cdots \lambda_{2 k}^{r_{2 k}} \lambda_{1}^{p_{1}} \cdots \lambda_{2 k}^{p_{2 k}}}
$$

where

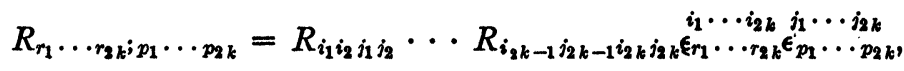

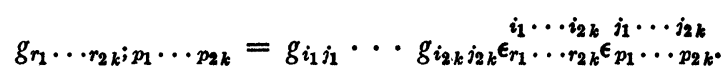

There are thus $[n / 2]$ such curvatures available to describe the nature of an $n$-space, the highest of which for $n$ even is $K_{T}$.

Very little is known about these curvatures; in particular it would be interesting to know the properties of a space which are implied by the vanishing of one or more of the curvatures $K_{2 k}$. For example, a four-dimensional space with $K_{2} \neq 0$ and with $K_{4}=0$ is still unexplored 
for the purposes of relativity theory. The difficulties involved in inventing such theorems arise partly from our lack of intuition when the dimension exceeds three and partly from the nonlinearity of the differential equations which these conditions imply.

6. The Hodge theory. Much more modern than Kronecker is the the work of Hodge [7] which has just begun to be exploited towards the ends with which I have been concerned today. Hodge has defined for skew-symmetric tensors two derived tensors which generalize the curl and divergence of a vector. For the tensor $T_{\alpha_{1}} \ldots \alpha_{r}$ (skew-symmetric in each pair of indices) these are:

$$
d T=T_{\alpha_{1} \cdots \alpha_{r}, \alpha_{r+1}} \epsilon^{\alpha 1 \cdots \alpha_{r+1} \alpha_{r+2} \cdots \alpha_{n}}
$$

and

$$
\delta T=T_{\alpha_{1} \cdots \alpha_{r}, \alpha_{r+1}} g^{\alpha_{r \alpha}+1} .
$$

If both $d T=0$ and $\delta T=0, T$ is called a harmonic tensor of order $r$. His main theorem is that the $r$ th Betti number of a closed orientable manifold $M^{n}$ is equal to the number of linearly independent harmonic tensors of order $r$ on $M^{n}$. This should lead to a method of computing the Betti number of such a manifold in terms of its local differential geometry. The only result so far is due to Bochner [4]: If the Ricci tensor $R_{j k} \equiv R_{i j k l} g^{i l}$ of a closed Riemann manifold is positive definite, the first Betti number of the manifold is zero. This result, however, was first proved in an entirely different fashion and in a somewhat stronger form by Myers [11].

\section{BIBLIOGRAPHY}

1. C. B. Allendoerfer, Rigidity for spaces of class greater than one, Amer. J. Math. vol. 61 (1939) pp. 634-644.

2. C. B. Allendoerfer and A. Weil, The Gauss-Bonnet theorem for Riemannian polyhedra, Trans. Amer. Math. Soc. vol. 53 (1943) pp. 101-129.

3. R. Beez, Zur Theorie der Krïmmungmasses von Mannigfaltigkeiten höherer Ordnung, Zeitschrift für Mathematik und Physik vol. 21 (1876) pp. 373-401.

4. S. Bochner, Vector fields and Ricci curvature, Bull. Amer. Math. Soc. vol. 52 (1946) pp. 776-797.

5. S. S. Chern, On the curvature integra in a Riemannian manifold, Ann. of Math. vol. 46 (1945) pp. 678-684.

6. S. Cohn-Vossen, Kitrzeste Wege und Totalkrimmung aus Flächen, Compositio Math. vol. 2 (1935) pp. 69-133.

7. W. V. D. Hodge, The theory and applications of harmonic integrals, Cambridge University Press, 1941. 39-49.

8. S. B. Myers, Riemannian manifolds in the large, Duke Math. J. vol. 1 (1935) pp. 
9. - Connections between differential geometry and topology, I, Duke Math. J. vol. 1 (1935) pp. 376-391.

10. - Connections between differential geometry and topology, II, Duke Math. J. vol. 2 (1936) pp. 95-102.

11. - Riemannian manifolds with positive mean curvature, Duke Math. J. vol. 8 (1941) pp. 401-404.

12. A. Weil, see Allendoerfer, C. B.

13. H. Weyl, Über die Starrheit der Eiflächen und konvexen Polyeder, Preuss. Acad. Wiss. Sitzungsber. (1917) pp. 250-266.

Haverford College 